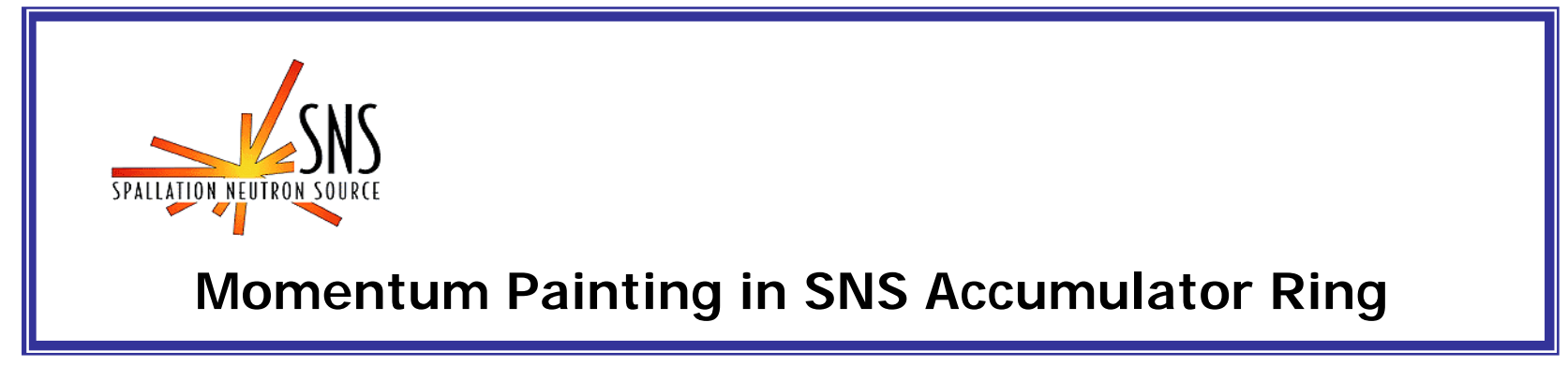

BNL/SNS TECHNICAL NOTE

NO. 124

M. Blaskiewicz

BNL, Upton, NY 11973, USA

August 25, 2003

COLLIDER-ACCELERATOR DEPARTMENT BROOKHAVEN NATIONAL LABORATORY

UPTON, NEW YORK 11973 


\title{
Momentum painting in SNS accumulator ring
}

\author{
M. Blaskiewicz, C-AD Brookhaven National Laboratory
}

August 25, 2003

For 2 MW operations, the peak beam current in the SNS accumulator ring approaches $100 \mathrm{~A}$. Collective effects are a significant concern and the various stability thresholds are monotonic functions of the momentum spread of the beam. Therefore, it is useful to have control of the injected momentum distribution. The energy corrector and spreader cavities were included in the design to allow the operations team to tune the rms energy spread of the injected beam, but these devices have been removed from the baseline. The energy corrector cavity runs at the nominal RF frequency. This device is located downstream of the main linac so that the longitudinal phase of the bunches is roughly proportional to their energy deviation. A proper choice of voltage corrects the centroid energy of the individual bunches. Next, the energy spreader cavity runs at a frequency that is offset from the noninal RF frequency by $\sim 100 \mathrm{kHz}$. This device provides a pseudo-random kick to the individual bunches which increases the rms energy spread of the beam without producing the large momentum tails associated with a debuncher cavity.

The energy corrector cavity is difficult to replace, since its operation depends of the correlation between phase and energy offset. On the other hand, the energy spreader cavity requires no drift. This opens the possibility of modifying the last accelerating cell of the superconducting linac for operation in energy spreader mode. Near the end of the supeconducting linac, each cavity increases the beam energy by $\approx 15 \mathrm{MeV}[1]$. The energy spreader cavity requires $\lesssim 5 \mathrm{MeV}$ so a single superconducting cavity could supply the necessary voltage. The mechanical tuners allow for $\approx 200 \mathrm{kHz}$ of frequency swing [2] and the $3 \mathrm{~dB}$ bandwidth of the klystrons is $\pm 1.3 \mathrm{MHz}$ [3]. Both of these are comfortably larger than the required $100 \mathrm{kHz}$ frequency offset.

The external Q of the fundamental power couplers is $7.0 \times 10^{5}$ [4] which gives a frequency offset of $f_{\text {res }} / 2 Q=575 \mathrm{~Hz}$ at the half power point. Therefore, modification of the fundamental power coupler is required.

\section{References}

[1] J. Stovall, J.H. Billen, S. Nath, H. Takaneda, L.M. Young, D. Jeon, K.R. Crandall, R. Shafer, PAC01, p 446 (2001).

[2] J. Preble et.al. , PAC03.

[3] S. Lenci, E. Eisen, B. Stockwell PAC03

[4] Y. Kang et al PAC01 p 1122 